\title{
Recurrent Falls: An Unusual Presentation of Spinal Meningioma in a Child
}

\author{
Cezar José Mizrahi' ${ }^{*}$, Eliel Ben-David ${ }^{2}$, Yakov Fellig ${ }^{3}$, MonyBenifla ${ }^{4}$ \\ ${ }^{1}$ Department of Neurosurgery, Hadassah-Hebrew University Medical Center, Jerusalem, Israel \\ ${ }^{2}$ Department of Radiology, Hadassah-Hebrew University Medical Center, Jerusalem, Israel \\ ${ }^{3}$ Department of Pathology, Hadassah-Hebrew University Medical Center, Jerusalem, Israel \\ ${ }^{4}$ The Pediatric Neurosurgery Unit, Department of Neurosurgery, Rambam Health Care Campus, Haifa, Israel
}

${ }^{*}$ Correspondence to: Cezar José Mizrahi, MD, Department of Neurosurgery, Hadassah-Hebrew University Medical Center, P.O. Box 12000 Jerusalem 91120, Israel; Telephone: +972 505172658; Email: Cezar@hadassah.org.il

Received: June 11, 2018; Accepted: June 22, 2018; Published: June 24, 2018;

\begin{abstract}
Spinal meningiomas in childhood are very rare. To the best of our knowledge, recurrent falls has not been previously described in this kind of pediatric tumor as an initial clinical presentation. We present an unusual case of a 13-year-old female with a history of recurrent falls for several months, who was diagnosed with spinal meningioma. After five months of the initial symptom, the patient presented with sudden onset paraparesis, urinary incontinence, and left lower limb hypoesthesia. An urgent MRI was performed revealing cervicothoracic junction meningioma. She underwent C6-T2 superiorly based laminotomy and complete resection of the tumor. Pathologic study revealed a benign transitional meningioma. Post-operative evolution was satisfactory, with full recover of muscular strength in the lower limbs and no new episodes of recurrent falls or of urinary incontinence. A follow-up MRI study, performed 3-months after surgery, demonstrated complete tumor resection with no signs of recurrence. We hypothesize that the patient initially developed recurrent falls due to dorsal cord compression, which resulted in a posterior column dysfunction and proprioception deficit. Recurrent falls may be an unusual presentation of posteriorly based spinal cord tumor.
\end{abstract}

Key words: meningioma - paraparesis - pediatric neurosurgery - recurrent falls - spinal meningioma

\section{Introduction}

Despite accounting for approximately $20 \%$ of all primary tumors in the central nervous system [1], meningiomas are very rare in pediatric patients, with an annual incidence of 8 cases per 1, 000, 000 people [2]. Meningiomas are generally benign and their recurrence is mostly related to the histologic type. Until now, approximately 60 cases of spinal meningiomas in childhood (SMCs) have been reported in the English literature. To the best of our knowledge, recurrent falls has not been previously described in this kind of pediatric tumor as an initial clinical presentation. The current standard of treatment for meningiomas in children is surgical resection [3]. We report a case of a 13-year-old female with a history of recurrent falls during several months as an initial presentation of a spinal meningioma.

\section{Case Material}

\section{Anamnesis, Physical Exam \& Imaging}

A 13-year-old female presented without any remarkable medical conditions except for a 5 month history of recurrent falls. She underwent a neurological exam by the pediatrician at the clinic, as well as head MRI and lower limbs CT that revealed no pathology. She presented to the emergency room with acute onset of paraparesis, urinary incontinence, and left lower limb hypoesthesia. Physical examination revealed bilateral reduction in muscle strength of the legs and thighs (both strength grade II/V), bilateral clonus, and bilateral positive pyramidal signs (Babinski, Chaddock, Oppenheim, and Gordon). Examination of the left lower limb demonstrated reduction of temperature and pain senses leveling at T3, with severe proprioception deficit of the left leg. Right side proprioception, temperature, pain and tactile examination were intact. Urgent spinal cord MRI with gadolinium-enhanced T1-weighted imaging, revealed a homogeneously enhancing intradural-extramedullary mass located posteriorly at the cervicothoracic junction area (C7-T1-T2) with a dural tail extending from the $\mathrm{C} 6$ to $\mathrm{T} 2$ levels (Fig.1 A, B). This mass exerted a local expansive-effect, creating medullar dorsal compression without significant peritumoral cord edema.

\section{Surgical procedure and follow-up}

In a prone position, with neurophysiological monitoring, a C6-T2 laminotomy was performed. The outer layer of dura matter was opened and the inner layer was attached to the tumor, a careful dissection was performed between the dural layers. The dura was opened at the lower and the upper ends of the tumor where it was free of disease. A good plain between tumor and the spinal cord was dissected. The tumor was lobulated, well-vascularized, with a hard rock consistency and multiple sites of calcification. Despite not finding a clear cleavage plane with the dura matter and an impressive compression of the spinal cord, an arachnoid plane was used and gross total tumor resection was 
achieved. The operative and post-operative courses were uneventful. The patient improved immediately after surgery, and returned to normal neurological status within 3 weeks. Paraffin embedded sections stained with H\&amp;E displayed a meningothelial neoplasm, with a partial fibrous pattern, and many tight whorls and psammoma bodies. No atypical features were detected. There was no necrosis and mitotic figures were not identified. On immunohistochemical stain the MIB-1 proliferation marker labeling index was about $2-3 \%$. The features are consistent with a pathological diagnosis of transitional meningioma, WHO grade I. (Figure 2). No risk factors for SCM as neurofibromatosis type 2 or exposure to radiation were found. A follow-up MRI study, 3-months after surgery, demonstrated complete tumor resection with no signs of recurrence (Figure $3 \mathrm{~A}, \mathrm{~B}$ ). She continue follow-up in our institution and 24 months after surgery the patient remains without neurologic deficit and no signs of tumor recurrence.

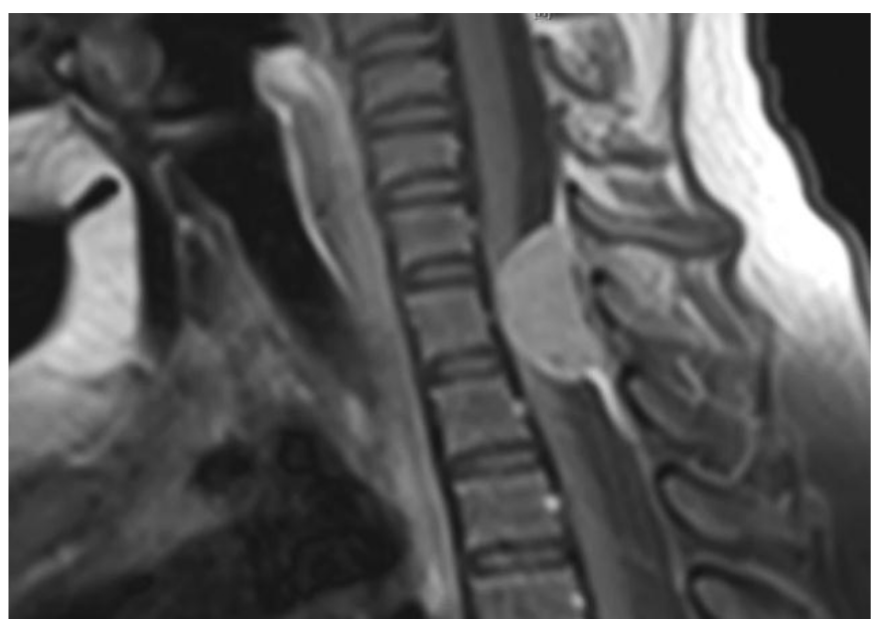

(1A) Sagittal

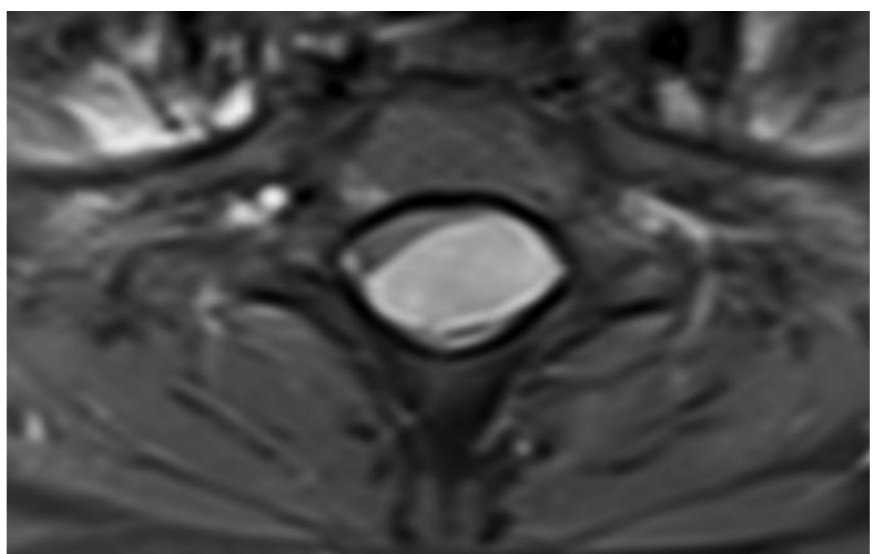

(1B) Axial

Figure 1. Sagittal (A) and axial (B) T1-weighted gadolinium-enhanced MRI showing a homogeneously enhancing intradural-extramedullary mass located posteriorly at the cervico-thoracic junction area (C7-T1-T2) with dural tail extending from the $\mathrm{C} 6$ to $\mathrm{T} 2$ levels, in a 13-year-old female with a history of recurrent falls.

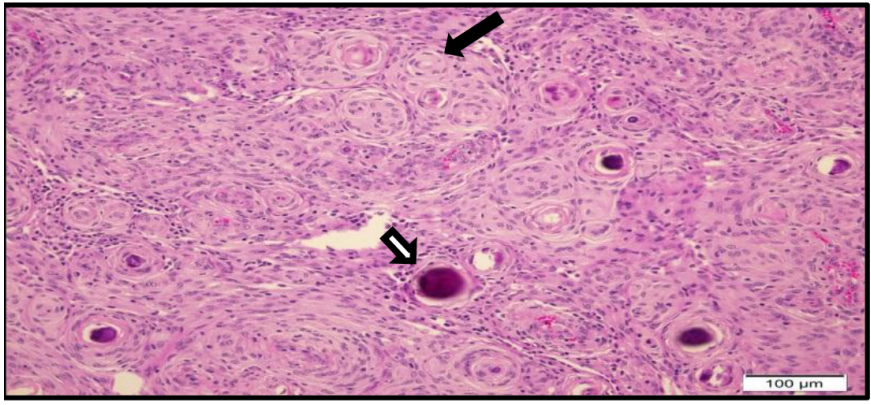

Figure 2. Paraffin embedded section stained with H\&E display a transitional meningioma, with many tight whorls (black arrow) and psammoma bodies (white arrow). No atypical features were detected. There is no necrosis and mitotic figures were not seen.

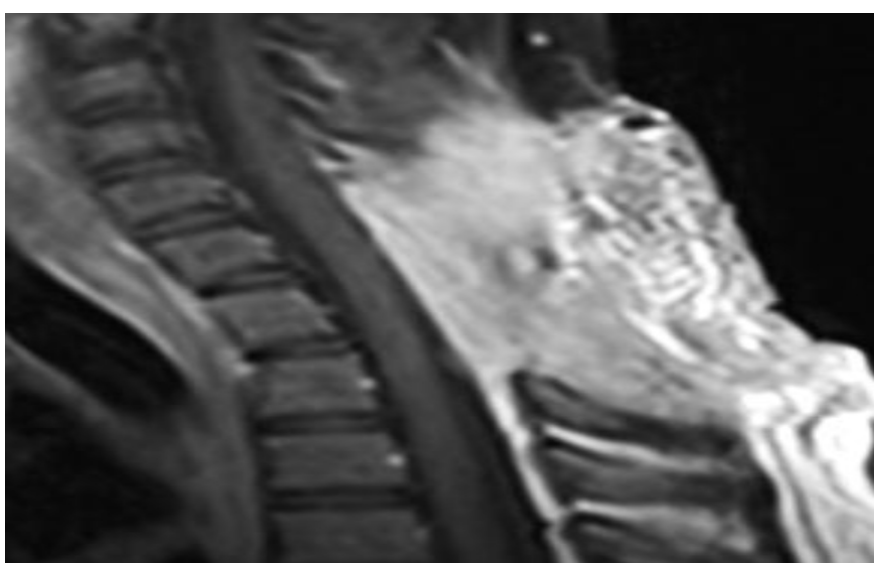

(3A) Sagittal

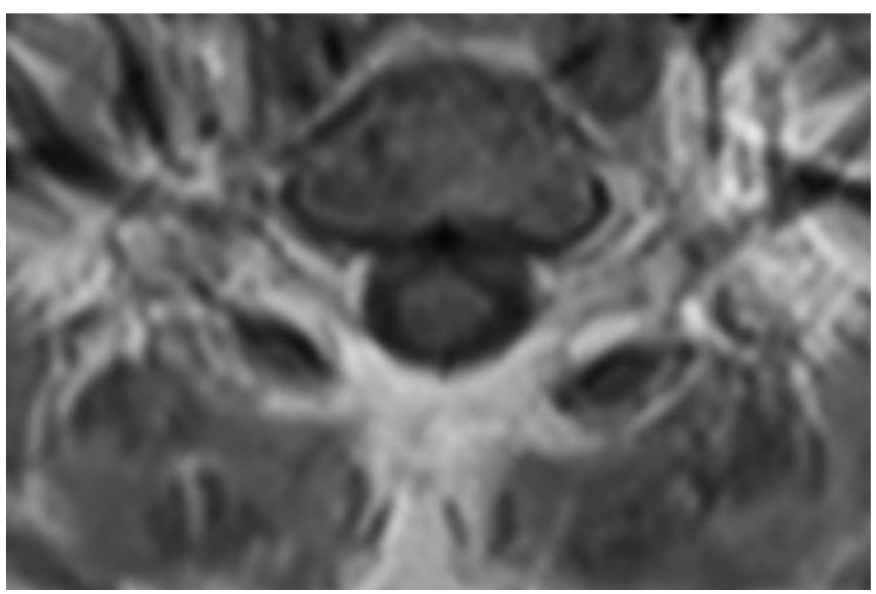

(3B) Axial

Figure 3. Sagittal (A) and axial (B) T1-weighted gadolinium-enhanced MRI at 3 month follow-up revealed complete tumor resection with no signs of recurrence. 


\section{Discussion}

We report a case of a 13-year-old female with a history of recurrent falls for several months as an initial presentation of a spinal meningioma at the level of the cervicothoracic junction. Recurrent falls as the only symptom, with a normal neurological exam, has not been previously reported in the English literature. We hypothesize that the initial neurological deficit was due to dorsal cord compression, resulting in a posterior column dysfunction and proprioception deficit, eventually leading to recurrent falls. Misdiagnosis resulted in a late diagnosis of a spinal meningioma in a child.

In the pediatric population, meningiomas account for less than $5 \%$ of brain tumors. Pediatric spinal tumors represent only $5 \%$ of tumors of the central nervous system, $25 \%$ of which occur in the intradural-extramedullary compartment [3] with an annual incidence of almost 1 per 1, 000, 000 children [4]. Reports from the adult population demonstrate that the majority of cases tend to occur in women (ratio around 2: 1). Nevertheless, this association is not replicated in children, where there are reports of an approximately 1.2: 1 predominance of cases in males [2]. In their retrospective analysis of 20 children, Greene at el. showed a median age of about 13 years at the time of the tumor presentation [2].

Clinical presentation of Spinal Meningiomas in Childhood (SMC) is variable, but consistent with the anatomical localization. The most common presenting symptoms are pain and limb weakness. Wang et al. [5] presented the largest series of SMC, with 10 patients treated from January 2002 to December 2010. They observed back pain in $60 \%$, followed by signs of limb weakness in $40 \%$, gait disturbance in $20 \%$, paresthesia in one patient, and urinary incontinence in another. Conesa D. et al. described a case of recurrent falls caused by spinal meningioma in an elderly woman [6]. Due to the slow growing pattern of meningioma, the symptoms develop gradually during 1-18 months [5].

The etiology of SMC is still not clear but some risk factors have been described, such as association with NF-2 and a history of radiation $[7,8]$. The link to NF-2 is becoming clearer, with the most common finding the loss of a tumor suppressor gene in the chromosome 22 (NF-2). Other gene mutations may contribute to the progression of the meningiomas, leading to the more aggressive, anaplastic type. Ionizing radiation is the environmental risk factor most predisposing to meningiomas [8]. The slight predominance of this tumor in women over men in adults raises the hypothesis of the influence of female hormones. Receptors for estrogen and progesterone have been suggested to cause faster tumor growth on the third trimester of pregnancy; however, the exact link between hormones and the development of meningiomas is still not clear [8].

Spinal cord meningioma is rare, with only 60 cases reported in the English literature. Reported ratios of intracranial to spinal meningiomas in children are 20:1 [12], 14: 1 [1], and 10: 1 [9]. From their review of 15 cases of SMCs, Colen et al. [13] reported that in 14 children the tumor was located in the thoracic and lumbar spinal canal. However, in Wang's et al. series [5] none of the tumors was in the lumbar area, with $50 \%$ in the cervical area, $30 \%$ thoracic, and $20 \%$ in the cervicothoracic junction.
The standard exam for diagnosing SMC is MRI study. The most common appearance in T1 and T2-weighted imaging is an extraaxial isointense mass. In the post-gadolinium injection, T1-weighted image, SMC appears as a homogeneously enhancing mass. Different degrees of peritumoral edema may be observed.

In 1993 the World Health Organization (WHO) ratified a new comprehensive classification of neoplasms affecting the central nervous system; its last edition (fifth) was published in 2016. According to the WHO classification, meningiomas are divided in to 3 grades - benign (grade I), atypical (grade II) and anaplastic or malignant (grade III). In the literature, psammomatous and fibroblastic meningiomas are the most common pathological subtypes reported in the largest series of pediatric [13] and adult [14] spinal meningiomas.

Gross total resection is the treatment of choice for SMC, with special care required to preserve the neurological function and stability of the growing spinal canal [14]. However, many factors should be considered in order to achieve a gross total resection, such as location, size, blood loss, adhesion, and the pathological subtypes (causing severe adhesion) [9]. Complete resection has been achieved with good post-operative outcome in most reported cases [5]. The extent of resection may also be predictive for recurrence.

In summary, spinal meningioma is a rare disease in the pediatric population. Not only pediatric neurosurgeons, but also pediatricians need be aware of this highly unusual presentation of SMC when facing recurrent falls in children. The diagnosis and treatment are relatively straightforward using an imaging study (MRI) and surgical tumor excision, respectively.

\section{Conflicts of Interest/Disclosure}

The authors declare that they have no financial or other conflicts of interest in relation to this research and its publication.

\section{References}

1. Rushing EJ, Olsen C, Mena H, Rueda ME, Lee YS, et al. (2005) Central nervous system meningiomas in the first two decades of life: a clinicopathological analysis of 87 patients. J Neurosurg 103: 489-495.

2. Greene S, Nair N, Ojemann JG, Ellenbogen RG, Avellino AM (2008) Meningiomas in children. Pediatr Neurosurg 44: 9-13. [crossref]

3. Binning M, Klimo P, Gluf W, Goumnerova L (2007) Spinal tumors in children. Neurosurg Clin N Am 18: 631-658. [crossref]

4. Kumar R, Giri PJ (2008) Pediatric extradural spinal tumors. Pediatr Neurosurg 44: 181-189. [crossref]

5. Wang XQ, Zeng XW, Zhang BY, Dou YF, Wu JS, et al. (2012) Spinal meningioma in childhood: clinical features and treatment. Childs Nerv Syst 28: 129-136. [crossref]

6. Conesa D, Ferrer A, Torres A, Formiga F, Pujol R, et al. (2013) An unusual and reversible cause of falls: spinal meningioma in an elderly woman. $J$ Am Geriatr Soc 61: 166-168. [crossref]

7. Mekitarian Filho E, Horigoshi NK, Carvalho WB, Hirscheimer MR, Bresolin AU, et al. (2010) Primary spinal meningioma in a 10-year-old boy. Arq Neuropsiquiatr 68: 804-806. [crossref]

8. Riemenschneider MJ, Perry A, Reifenberger G (2006) Histological classification and molecular genetics of meningiomas. Lancet Neurol 5: 1045-1054

9. Campbell BA, Jhamb A, Maguire JA, Toyota B, Ma R (2009) Meningiomas in 2009: controversies and future challenges. Am J Clin Oncol 32: 73-85. [crossref]

10. Hanel RA, Tatsui CE, Araujo JC, Grande CV, Antoniuk A, et al. (2001) [Meningiomas in pediatric patients: report of 2 cases]. Arq Neuropsiquiatr 59: 623-627. [crossref]

11. Watanabe M, Chiba K, Matsumoto M, Maruiwa H, Fujimura Y, et al. (2001) Infantile spinal cord meningioma. Case illustration. J Neurosurg 94: 334. [crossref] 
12. Colen CB, Rayes M, McClendon J Jr, Rabah R, Ham SD (2009) Pediatric spinal clear cell meningioma. Case report. J Neurosurg Pediatr 3: 57-60. [crossref]

13. Loh JK, Lin CK, Hwang YF, Hwang SL, Kwan AL, et al. (2005) Primary spinal tumors in children. J Clin Neurosci 12: 246-248. [crossref]
14. Engelhard HH, Villano JL, Porter KR, Stewart AK, Barua M, et al. (2010) Clinical presentation, histology, and treatment in 430 patients with primary tumors of the spinal cord, spinal meninges, or cauda equina. J Neurosurg Spine 13: 67-77. [crossref]

\section{Citation:}

Mizrahi CJ, Ben-David E, Fellig Y, Benifla M (2018) Recurrent falls: An Unusual Presentation of Spinal Meningioma in a Child. J Neurol Neurocrit Care Volume 1(1): 1-4. DOI: 10.31038/JNNC.1000105 